\title{
Feasibility of spiral fMRI based on an LTI gradient model
}

\author{
Nadine N. Graedel ${ }^{\mathrm{a}, \mathrm{b}}$, Lars Kasper ${ }^{\mathrm{b}, \mathrm{c}}$, Maria Engel ${ }^{\mathrm{b}}$, Jennifer Nussbaum ${ }^{\mathrm{b}}$, Bertram J. Wilm ${ }^{\mathrm{b}}$, \\ Klaas P. Pruessmann ${ }^{\mathrm{b}}$, S. Johanna Vannesjo ${ }^{\mathrm{a}, *}$ \\ ${ }^{a}$ Wellcome Centre for Integrative Neuroimaging, FMRIB, Nuffield Department of Clinical Neurosciences, University of Oxford, Oxford, United Kingdom \\ ${ }^{\mathrm{b}}$ Institute for Biomedical Engineering, ETH Zurich and University of Zurich, Zurich, Switzerland \\ ${ }^{\mathrm{c}}$ Translational Neuromodeling Unit, Institute for Biomedical Engineering, University of Zurich and ETH Zurich, Zurich, Switzerland
}

\section{A R T I C L E I N F O}

\section{Keywords:}

Functional MRI

High-resolution fMRI

Spiral imaging

Single-shot spiral

Linear time-invariant

GIRF

\begin{abstract}
A B S T R A C T
Spiral imaging is very well suited for functional MRI, however its use has been limited by the fact that artifacts caused by gradient imperfections and $\mathrm{B}_{0}$ inhomogeneity are more difficult to correct compared to EPI. Effective correction requires accurate knowledge of the traversed k-space trajectory. With the goal of making spiral fMRI more accessible, we have evaluated image reconstruction using trajectories predicted by the gradient impulse response function (GIRF), which can be determined in a one-time calibration step.

GIRF-predicted reconstruction was tested for high-resolution $(0.8 \mathrm{~mm})$ fMRI at 7T. Image quality and functional results of the reconstructions using GIRF-prediction were compared to reconstructions using the nominal trajectory and concurrent field monitoring.

The reconstructions using nominal spiral trajectories contain substantial artifacts and the activation maps contain misplaced activation. Image artifacts are substantially reduced when using the GIRF-predicted reconstruction, and the activation maps for the GIRF-predicted and monitored reconstructions largely overlap. The GIRF reconstruction provides a large increase in the spatial specificity of the activation compared to the nominal reconstruction.

The GIRF-reconstruction generates image quality and fMRI results similar to using a concurrently monitored trajectory. The presented approach does not prolong or complicate the fMRI acquisition. Using GIRF-predicted trajectories has the potential to enable high-quality spiral fMRI in situations where concurrent trajectory monitoring is not available.
\end{abstract}

\section{Introduction}

Blood Oxygen Level Dependent (BOLD) functional magnetic resonance imaging (fMRI) requires fast imaging, for which acquisitions with Echo Planar Imaging (EPI) readouts are currently used as the gold standard. Spiral readouts (Ahn et al., 1986) have many desirable properties for rapid acquisitions and have long been considered a promising alternative to EPI for fMRI (Glover, 2012): They can provide higher k-space sampling efficiency compared to EPI sampling (Glover, 2012; Glover and Lee, 1995; Noll et al., 1995), translating into higher resolution within a given readout time, and they also allow for a more flexible choice of echo time (TE). The combination is especially useful for high-resolution fMRI at 7T or above, where for EPI large parallel imaging factors or Partial Fourier are required to achieve the optimal TE for BOLD contrast. Further, spiral imaging has naturally reduced sensitivity to pulsatile motion (Glover and Lee, 1995; Yang et al.,
1998), and spiral-in/out trajectories (Glover and Law, 2001) can improve fMRI in regions prone to dropout, such as the orbitofrontal cortex. Finally, spiral sampling is more amenable to high undersampling factors, as the point spread function results in relatively incoherent aliasing (Wright et al., 2014), which can be less detrimental to image quality compared to coherent aliasing, which occurs in undersampled EPI.

Despite these advantages spiral imaging has not yet become a mainstream fMRI acquisition strategy. The reasons for the slow uptake of spiral fMRI include the fact that artifacts caused by gradient imperfections (discrepancy between the actual and nominal gradients) and $\mathrm{B}_{0}$ inhomogeneities are more difficult to correct for spiral trajectories compared to EPI. Localized off-resonance resulting from susceptibilityinduced field inhomogeneities cause dropout and shifts in EPI, for which a number of established correction methods exist (Andersson et al., 2003; Smith et al., 2004). Similarly for gradient infidelity, the correction

\footnotetext{
* Corresponding author.

E-mail address: johanna.vannesjo@gmail.com (S.J. Vannesjo).
} 
of the Nyquist ghost artifact in EPI is considered a routine step in image reconstruction, for example using navigator lines acquired before the readout (Schmitt et al., 1998). For spiral readouts, $B_{0}$ inhomogeneities and gradient imperfections cause blurring, geometric distortions and dropout. To improve gradient fidelity, it is common to perform a delay correction in spiral imaging (Bhavsar et al., 2014; Börnert et al., 1999; Robison et al., 2010). However this typically requires extra calibration scans, and is usually not as effective as the EPI delay correction. Moreover, unlike EPI, the effects of $\mathrm{B}_{0}$ inhomogeneities are difficult to correct via image processing methods, and are therefore commonly not addressed at all. As a result, spiral fMRI images have often been blurry, especially around the air-tissue interfaces in the frontal sinuses and ear canals.

Monitoring of the encoding fields during the acquisition using Nuclear Magnetic Resonance (NMR) field probes (Barmet et al., 2008; De Zanche et al., 2008) allows precise measurement of the traversed $\mathrm{k}$-space trajectory. In conjunction with a $\mathrm{B}_{0}$ field map covering the imaging FOV, included in a model-based reconstruction, this has been shown to yield high-quality spiral imaging (Engel et al., 2018; Kasper et al., 2018; Wilm et al., 2016), enabling high-resolution spiral fMRI (Kasper et al., 2019). However, the concurrent monitoring requires an additional hardware setup, which is not always available. One alternative to direct field measurements is to model the behavior of the gradient chain. With the appropriate model, deviations from the prescribed encoding that are reproducible (for example, induced by eddy currents) can be measured and corrected for. It has previously been shown (Addy et al., 2012; Vannesjo et al., 2013) that the gradient chain can be considered as a linear, time invariant (LTI) system to a high degree of accuracy. For an LTI system, the relation between the input to the system and its output is determined by the impulse response of the system - in the case of the gradient chain, the gradient impulse response function (GIRF). The GIRF of a specific system can be characterized in a one-time calibration procedure, which then enables to predict the actual gradient output to arbitrary input pulses. The GIRF-predicted output can be used as basis for image reconstruction, which has been shown to yield high quality images for a range of different trajectories (Addy et al., 2012; Campbell-Washburn et al., 2016; Vannesjo et al., 2016).

We have previously demonstrated that GIRF-prediction enables single-shot spiral images with only minor quality differences to using the monitored trajectory (Vannesjo et al., 2016). This evaluation was performed on individual images. In fMRI, however, we perform high duty cycle imaging over extended periods of time (5-10 min for a typical fMRI run with a single fMRI session often containing multiple runs). We know that there are long-term effects, for example gradient heating, that violate the LTI assumption at the basis of the GIRF prediction. But we do not know to what extent this will affect an image time-series, such as required for fMRI. We also do not know how the resulting imperfections propagate into the fMRI analysis. The aim of the present work is to evaluate the utility of GIRF-based reconstruction for spiral functional MRI. The results are assessed by comparison with reconstructions based on concurrent field monitoring and nominal trajectories with gradient delay correction.

\section{Methods}

All data were acquired on a 7T Achieva system (Philips Healthcare, Best, Netherlands) using a quadrature-transmit coil and 32-channel head receive array (Nova Medical, Wilmington, MA). The manufacturer's built-in eddy current compensation was kept activated for all experiments.

\subsection{LTI gradient model}

A linear and time-invariant system can be described via its impulse response function, which is the output of the system to a very brief in- put pulse. Knowledge of the system's impulse response allows predicting the system response $o(t)$ to any input, via convolution of the input waveform $i(t)$ with the impulse response $h(t)$. In the frequency domain this corresponds to a multiplication with the transfer function $H(\omega)$, i.e. the Fourier transform of the impulse response:

$o(t)=\int_{-\infty}^{\infty} i(\tau) \cdot h(t-\tau) d \tau \stackrel{F T}{\leftrightarrow} O(\omega)=I(\omega) \cdot H(\omega)$

The characterization of the gradient chain was performed similarly as described by Vannesjo et al. (2013): A set of gradient input pulses were played out and the resulting magnetic fields were measured with a dynamic field camera (Dietrich et al., 2016a) consisting of $16{ }^{1} \mathrm{H}$ NMR field probes distributed on the surface of a sphere of $10 \mathrm{~cm}$ radius. Spherical harmonic basis functions up to the 3rd order were fitted to the probe measurements. The GIRF was calculated via frequency-domain division of the measured output by the known inputs, using least-squares combination of data from different input pulses. For an accurate GIRF calibration the input gradient pulses should cover the entire range of expected frequencies, while complying with hardware and acquisition time constraints. This was achieved by using 12 different triangular pulses (slew rate $200 \mathrm{~T} / \mathrm{m} / \mathrm{s}$, time-to-peak $20-158 \mathrm{~ms}$ at $\sim 12$-ms increments). The GIRF measurements took approximately 3 min (12 gradient pulses, 3 gradient directions, 4 averages, $1.2 \mathrm{~s} \mathrm{TR}$ ). The individual probe signals were corrected for concomitant fields terms which are a known deviation from the LTI assumption (for details see (Vannesjo et al., 2016)). This correction was also applied to the concurrent field monitoring data described in the next section.

The LTI model of the gradient system was subsequently used to estimate actual gradient time courses of the imaging acquisitions through a frequency-domain multiplication of the nominal gradient with the measured gradient transfer function (see Suppl. Fig. 1. for the measured transfer function).

\subsection{Concurrent field monitoring}

Concurrent field monitoring was performed during all imaging acquisitions using ${ }^{19} \mathrm{~F}$ NMR field probes positioned between the transmit coil and the 32-channel receive array (as shown in (Engel et al., 2018), Fig. 1). The field probes were excited before the start of the readout gradient and the probe signal was acquired concurrently with the imaging readout. Due to the long readout and the strong imaging gradients, the probe signal can de-phase prior to the end of the monitoring period. Each probe's signal was therefore visually inspected and if the probe signal had very low amplitude and the signal phase exhibited discontinuities the probe was excluded from the spherical harmonic fit. Per subject, between 5 and 7 probes (on average 6.2) were excluded in this study, leaving approximately 10 probes for the fit.

The data from the remaining probes was fitted to up to 1 st order spherical harmonics producing linear gradient field terms in the three orthogonal directions (and the corresponding $k$-space trajectory $\mathrm{k}_{\mathrm{x}}, \mathrm{k}_{\mathrm{y}}, \mathrm{k}_{\mathrm{z}}$ ), as well as a 0th-order field term (and the corresponding phase term, $\mathrm{k}_{0}$ ), which reflects global field changes over time.

Undesired saturation of the NMR field probe signal can occur if the repetition time of the probe excitation is short relative to the $T_{1}$ of the probes. To allow sufficient time for signal recovery between measurements, the field camera recording was performed on every third slice. For the non-monitored slices the k-space trajectory from the last monitored slice was used.

\section{3. fMRI acquisition}

The raw coil data and concurrently monitored trajectories analyzed in this work were acquired as part of a recent study exploring the use of concurrently monitored single-shot spirals for fMRI (Kasper et al., 2019). The dataset contains acquisitions from seven healthy volunteers who 

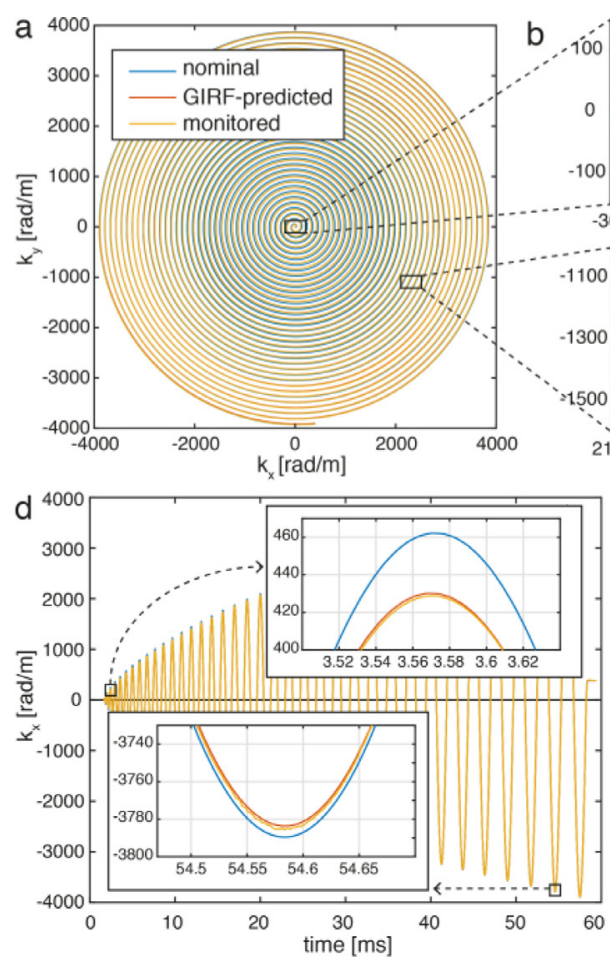
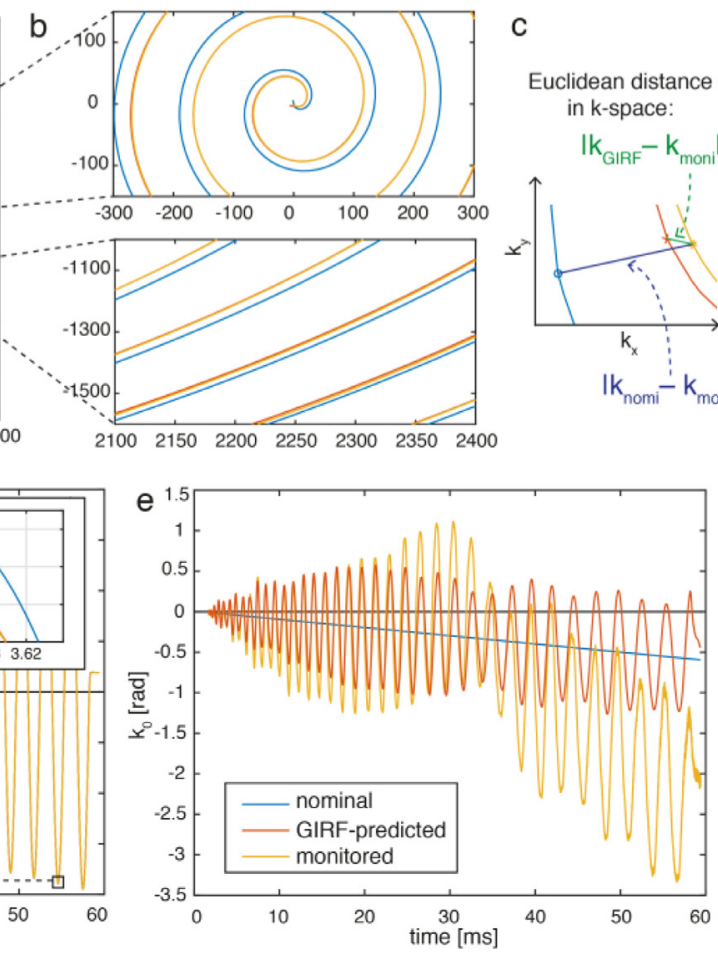

c
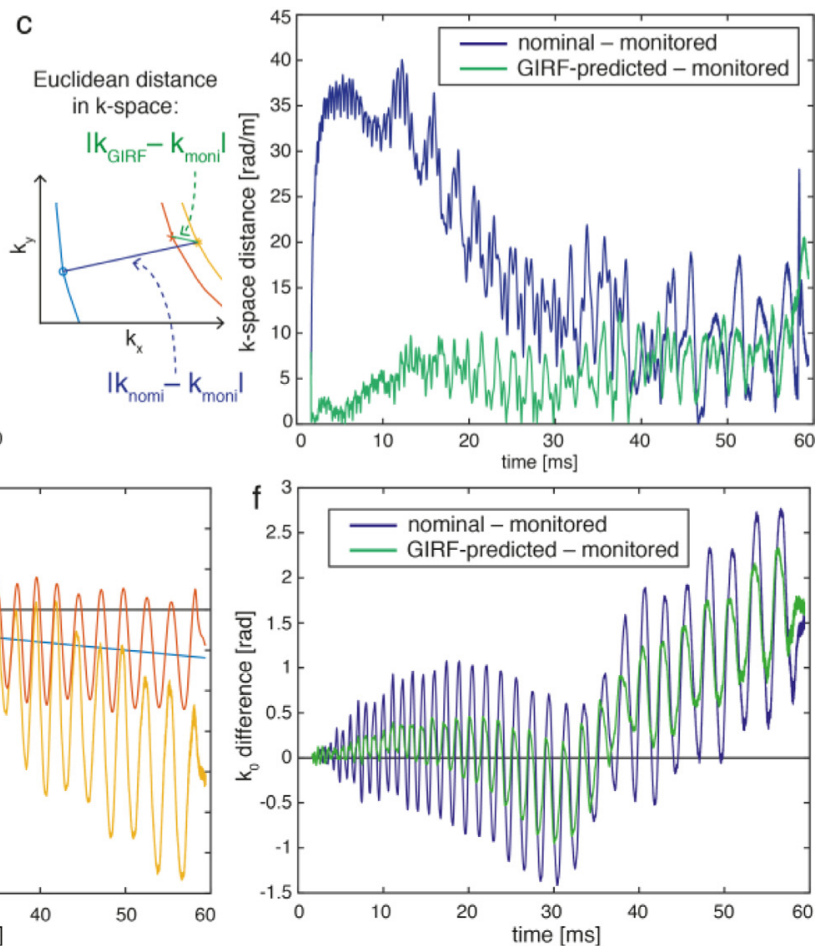

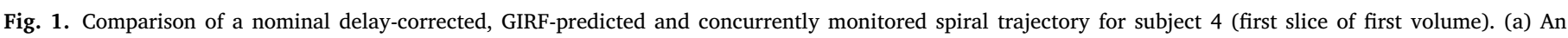

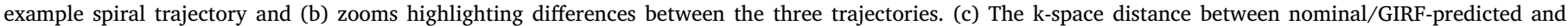

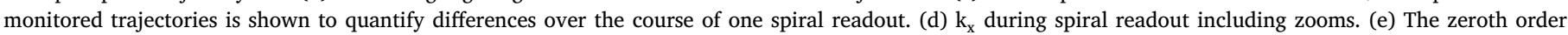

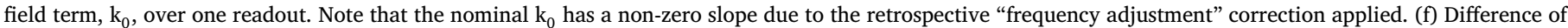
nominal/GIRF-predicted to measured $\mathrm{k}_{0}$.

had giving written informed consent and were scanned with approval of the local ethics committee. The visual fMRI paradigm used a simple retinotopic mapping protocol (Warnking et al., 2002). It was designed to stimulate quarter-fields of the visual cortex, similar to the one used in Kasper et al. (2014). The subjects were presented with 15 s blocks of two flickering, black-and-white checkerboard-filled $90^{\circ}$ wedges separated by $180^{\circ}$, interleaved with $15 \mathrm{~s}$ of rest (fixation cross). Alternating blocks of upper left/lower right (ULLR) and blocks of upper right/lower left (URLL) wedges were presented over 100 volumes ( 330s). The subjects were instructed to fixate on a point at the center between the wedges. In order to maintain the subjects' attention, they were asked to respond to any contrast alteration of the fixation point via a button box.

Images were acquired with a multi-slice $2 \mathrm{D}$ gradient-echo sequence with a single-shot Archimedean spiral-out readout (designed according to Lustig et al. (2008)) of $57 \mathrm{~ms}$ duration. The radial spacing of samples was chosen to undersample k-space by a factor of 4 with respect to the field-of-view (FOV) of $23 \mathrm{~cm}$. The transversal images were acquired with an in-plane resolution of $0.8 \mathrm{~mm}$ isotropic and a TE of $20 \mathrm{~ms}$, selected for good BOLD contrast. 36 slices of $0.89 \mathrm{~mm}$ thickness (with a slice gap of $0.11 \mathrm{~mm}$ ) were acquired, resulting in a FOV of $23 \times 23 \times 3.6 \mathrm{~cm}$ and a volume TR of $3.3 \mathrm{~s}$. Excitations were preceded by a Spectral Presaturation with Inversion Recovery (SPIR) fat suppression module (Kaldoudi et al., 1993).

Measurements from five optically connected temperature sensors (cast into the epoxy that structurally supports the gradient coil) were used to monitor gradient coil temperature with a temporal resolution of $\sim 18 \mathrm{~s}$. The data was used for evaluation of the temperature change over scans and sessions, and was compared to the image RMSE over time for an example subject. Because the temperature recordings were not synchronized with the imaging scans, there was some ambiguity in the relative timing of the two. Approximate alignment was performed based on time stamps in log files, and further adjusted within $+/-10 \mathrm{~s}$ by visually aligning the temperature data with the image RMSE curve. Due to the timing ambiguity we refrained from a full quantitative analysis of the relationship between trajectory/imaging errors and temperature changes.

A Cartesian multi-echo GRE scan (FOV $=23 \mathrm{~cm}$, resolution $=1 \mathrm{~mm}$ isotropic, $\mathrm{TE}_{1}=4 \mathrm{~ms}, \Delta \mathrm{TE}=1 \mathrm{~ms}, 6$ echoes) was collected to estimate coil sensitivities and $B_{0}$ maps. The first echo was used to estimate the coil sensitivities, by dividing the single-coil images by the root-sum-ofsquares coil combination. The $\mathrm{B}_{0}$ maps were calculated by voxel-wise fitting of the signal phase over the different echoes. Both the coil sensitivity maps and the $\mathrm{B}_{0}$ maps were spatially smoothed before use in subsequent image reconstructions.

One subject was excluded from further analysis due to reduced signal in multiple channels of the head receive array. The data from the six remaining volunteers were reconstructed and analyzed as described below.

\subsection{Image reconstruction and fMRI analysis}

The images were reconstructed offline in Matlab (MathWorks, Natick, MA, USA) using CG-SENSE (Pruessmann et al., 2001) with multifrequency interpolation for fast off-resonance correction (Man et al., 1997; Sutton et al., 2003). The full reconstruction model takes into account coil sensitivities, the static field as well as field dynamics over time (for details see (Engel et al., 2018; Kasper et al., 2019)). For each data set three reconstructions were performed using the following $\mathrm{k}$-space trajectories:

1 Delay-corrected nominal trajectory (labeled nominal in figures) 2 GIRF-predicted trajectory (labeled GIRF or GIRF-predicted in figures) 
3 Trajectory measured with concurrent field monitoring (labeled monitored in figures).

For the monitored and GIRF-predicted reconstructions the imaging data were demodulated by the measured/predicted 0th-order phase terms $\mathrm{k}_{0}$. It has previously been demonstrated that demodulation with an accurate estimate of $\mathrm{k}_{0}$ can substantially improve image quality (Vannesjo et al., 2016).

A center frequency adjustment is a typical fMRI pre-scan, which was not performed in this study because it is redundant when using concurrent field monitoring. In order to not artificially disadvantage the GIRF-predicted and nominal reconstructions, we performed a processing step equivalent to frequency adjustment. The center frequency was determined once for each fMRI time series via a linear fit on the first $0.9 \mathrm{~ms}$ of the monitored $\mathrm{k}_{0}$ (using the data from first slice of the first volume, before the readout gradient starts). The imaging data for the entire fMRI time series was then demodulated by this center frequency for the nominal and the GIRF-predicted reconstruction.

The nominal trajectories were delay-corrected prior to reconstruction. Delay calibration was performed by minimizing the RMSE trajectory difference between the nominal and the monitored trajectory. The delay calibration was performed on the first readout of the time series of all participants, and the mean over the participants was taken to be the delay. The calibrated delay was then kept fixed for all readouts and for all participants. A global delay for all gradient axes was chosen as the small differences between the gradient axes were within the standard deviation of the delay calibration.

The reconstructed image time series was corrected for subject translations and rotations using MCFLIRT (Jenkinson et al., 2002) in the FMRIB Software Library (FSL) (Jenkinson et al., 2012) and was prewhitened using FILM/FSL (Woolrich et al., 2001). The GLM (analyzed using FEAT/FSL (Jenkinson et al., 2012)) contained the regressors for the ULLR and the URLL stimulation blocks convolved with a Gamma function. Activation was assessed using z-statistics contrasting ULLR versus URLL ([1 -1] in the design matrix). In order to produce fMRI data with high spatial specificity we performed no spatial smoothing or clustering. We report activation maps at a liberal threshold of $\mathrm{z}>2.3$ $(p<0.01)$.

All of the analysis was performed on a per-subject basis in the space of each subject's functional data to avoid any degradation of the spatial resolution by registration. The first echo of the multi-echo GRE scans was registered to the functional data and used as the subjects' structural image. For the analysis of the functional results, masks of the grey matter (GM) and white matter (WM) in the visual cortex were determined as an intersection of a V1-V3 mask (using Juelich atlas (Schleicher et al., 2005; Zilles and Amunts, 2010) labels 81-86 in FSL) and subject-specific GM/WM masks generated by segmenting the structural image using FAST/FSL (Zhang et al., 2001). The GM/WM masks were generated by conservatively thresholding the partial volume maps at 0.8 to exclude most partial volume voxels from the masks. In Table 1 the average and 90th percentile of the absolute value of significant $(z>2.3)$ z-stats within the GM V1-V3 ROI are reported.

The concurrently monitored trajectories and the resulting image reconstructions were used as reference to assess the nominal and GIRFpredicted data. The trajectory error and image artifacts were accordingly quantified as root-mean-squared error (RMSE) compared to concurrent monitoring (Table 1), and the GIRF/nominal z-statistic maps were compared to the ones derived from concurrent monitoring data. Additionally, receiver operator characteristic (ROC) curves were used to provide an assessment of the fMRI results without selecting the monitored reconstruction as the ground truth. ROC analysis typically involves plotting the number of true-positives against the number of falsepositive findings. In this work we used the subject-specific gray and white matter masks of V1-V3 to identify "true positive" and "false positive" activation respectively and plotted this while varying the z-statistic threshold from 0 to the maximum $\mathrm{z}$ present in the data. The area under the curve (AUC) gives a measure of spatial specificity and was used to compare between the reconstructions. This analysis has two major advantages: i) it provides a quantitative assessment of the activation maps without requiring one reconstruction as ground truth, and ii) it encompasses the full z-statistics instead of relying on a specific significance threshold (for example $z>2.3$ ).

The temporal SNR (tSNR) was evaluated in the motion-corrected fMRI time series, and was calculated on a voxel-by-voxel basis as the mean signal over time divided by the temporal standard deviation of the signal. The tSNR was averaged over the GM V1-V3 ROI and reported in Table 1 for each subject and reconstruction.

\section{Results}

The delay-corrected nominal spiral trajectories deviate substantially from the ones measured with the NMR field probes, especially close to the center of k-space where the gradients are rapidly changing (Fig. 1 a-d). For the example subject shown in Fig. 1 the distance between the nominal and the measured trajectories reaches $40.1 \mathrm{rad} / \mathrm{m}$ which corresponds to $\sim 1.5^{*} \Delta \mathrm{k}\left(\Delta \mathrm{k}=2 \pi^{*} 1 / \mathrm{FOV}\right)$. The GIRF-predicted spiral trajectories follow the measured ones much more closely, especially during the first $40 \mathrm{~ms}$ of the readout. Towards the edge of k-space, however, there is little improvement from the GIRF-predicted trajectories over the nominal ones. For the example subject in Fig. 1 the maximum k-space deviation between the GIRF-predicted and the measured trajectories is $20.5 \mathrm{rad} / \mathrm{m}$, which corresponds to $\sim 0.75^{*} \Delta \mathrm{k}$, with the largest absolute deviations occurring at the end of the readout.

The root-mean-square trajectory error (RMSE), defined here as the Euclidian distance to the monitored trajectory, averaged over all slices and volumes, is reported for all subjects in Table 1. Averaged over all subjects, the nominal RMSE and GIRF-predicted RMSE were 19.66 $\mathrm{rad} / \mathrm{m}$ and $10.85 \mathrm{rad} / \mathrm{m}$, respectively. Fig. 1e/f show the concurrently monitored, GIRF-predicted and nominal $\mathrm{k}_{0}$, reflecting global temporal variations in $\mathrm{B}_{0}$. The measured $\mathrm{k}_{0}$ oscillations are closely coupled with those of the spiral readout gradients. This is partially predicted by the GIRF, but the amplitude of oscillations is not captured accurately, especially towards the end of the readout. Additionally, the monitored $\mathrm{k}_{0}$ exhibits slower trends over the readout, namely a change in slope at about $35 \mathrm{~ms}$ (see monitored $\mathrm{k}_{0}$ (yellow line) in 1e). These slower dynamics are qualitatively similar across subjects and have also been observed in other studies acquired on the same MRI scanner (Engel et al., 2018). The reason for this change in slope is not yet know but it is not captured by the GIRF prediction, suggesting that it is not linearly related to the gradient waveform. Average RMSE over all subjects (see Table 1 for results for individual subjects) is $1.06 \mathrm{rad}$ for nominal $\mathrm{k}_{0}$ and $0.94 \mathrm{rad}$ for GIRF-predicted $\mathrm{k}_{0}$

The GIRF-predicted and nominal trajectories are the same for all reconstructions within a time series, whereas the monitored trajectory is updated every 3rd readout. Fig. 2 illustrates how the monitored trajectory changes over the course of the fMRI experiment. Over the $5.5 \mathrm{~min}$ experiment the trajectory gradually shifts from the first volume (blue) to the last volume (red). Note that the shift, however, is small compared to the distance to the nominal trajectory. The RMSE for the GIRFpredicted vs. monitored trajectories increases over the course of the experiment, but remains considerably below the RMSE of the nominal vs. measured trajectories (Fig. 2b). Interestingly, the latter slightly improves during the experiment. For the measured $\mathrm{k}_{0}$ (Fig. $2 \mathrm{c} / \mathrm{d}$ ) the amplitude of the observed oscillations remains fairly consistent but the slope of the $\mathrm{k}_{0}$ drift over the readout changes substantially. Most variations over the acquisition are likely caused by gradient heating. For our acquisition we observed gradient temperature increases of $\sim 10-15^{\circ} \mathrm{C}$ over a single $\sim 5 \mathrm{~min}$ fMRI run and $\sim 25-30^{\circ} \mathrm{C}$ increase over the entire scanning session consisting of four fMRI runs and a number of shorter scans. 
Table 1

Results summary metrics for all subjects (from top to bottom): RMSE trajectory errors for the k-space distance and the zeroth order field term, $\mathrm{k}_{0}$, using the monitored trajectory as the ground truth, RMSE image error using the monitored reconstruction as the reference, average tSNR in the brain, average and 90th percentile of significant z-statistics in the GM V1-V3 ROI and the AUC from the ROC plots to evaluate spatial specificity of the z-statistic maps.

\begin{tabular}{lllllllll}
\hline & Subjects $\rightarrow$ & 1 & 2 & 3 & 4 & 5 & 6 & Mean \\
\hline \multirow{2}{*}{ Trajectories } & RMSE k-space distance nomi [rad/m] & 19.78 & 19.60 & 20.84 & 19.63 & 18.67 & 19.40 & 19.66 \\
& RMSE k-space distance GIRF [rad/m] & 9.85 & 10.66 & 12.80 & 9.07 & 10.55 & 12.19 & 10.85 \\
& RMSE k $_{0}$ nomi [rad] & 1.19 & 0.89 & 1.08 & 1.04 & 1.34 & 0.85 & 1.06 \\
\multirow{3}{*}{ Images } & RMSE k GIRF [rad] & 1.11 & 0.75 & 0.90 & 0.84 & 1.24 & 0.81 & 0.94 \\
& RMSE nomi [\%] & 6.34 & 6.45 & 5.22 & 6.97 & 5.03 & 5.06 & 5.85 \\
tSNR & RMSE GIRF [\%] & 2.01 & 2.07 & 1.98 & 2.36 & 2.52 & 1.95 & 2.15 \\
& tSNR moni & 16.44 & 19.39 & 14.98 & 15.43 & 13.95 & 15.35 & 15.93 \\
& tSNR GIRF & 14.94 & 18.25 & 14.23 & 14.71 & 13.37 & 14.67 & 15.03 \\
fMRI & tSNR nomi & 15.47 & 19.42 & 14.90 & 15.66 & 14.09 & 15.46 & 15.84 \\
& mean zstat moni & 3.74 & 4.09 & 3.98 & 4.33 & 4.04 & 3.72 & 3.98 \\
& mean zstat GIRF & 3.66 & 3.92 & 3.86 & 4.14 & 3.73 & 3.75 & 3.84 \\
& mean zstat nomi & 3.54 & 3.94 & 3.85 & 3.84 & 3.55 & 3.81 & 3.76 \\
& 90th perct. moni & 5.72 & 6.74 & 6.34 & 7.13 & 6.62 & 5.76 & 6.39 \\
& 90th perct. GIRF & 5.58 & 6.35 & 6.04 & 6.74 & 5.77 & 5.88 & 6.06 \\
& 90th perct. nomi & 5.14 & 6.20 & 5.83 & 5.97 & 5.32 & 5.94 & 5.73 \\
& ROC AUC moni & 928 & 758 & 860 & 1062 & 624 & 755 & 831 \\
& ROC AUC GIRF & 809 & 615 & 703 & 981 & 577 & 723 & 735 \\
& ROC AUC nomi & 548 & 341 & 266 & 295 & 80 & 463 & 332 \\
\hline
\end{tabular}
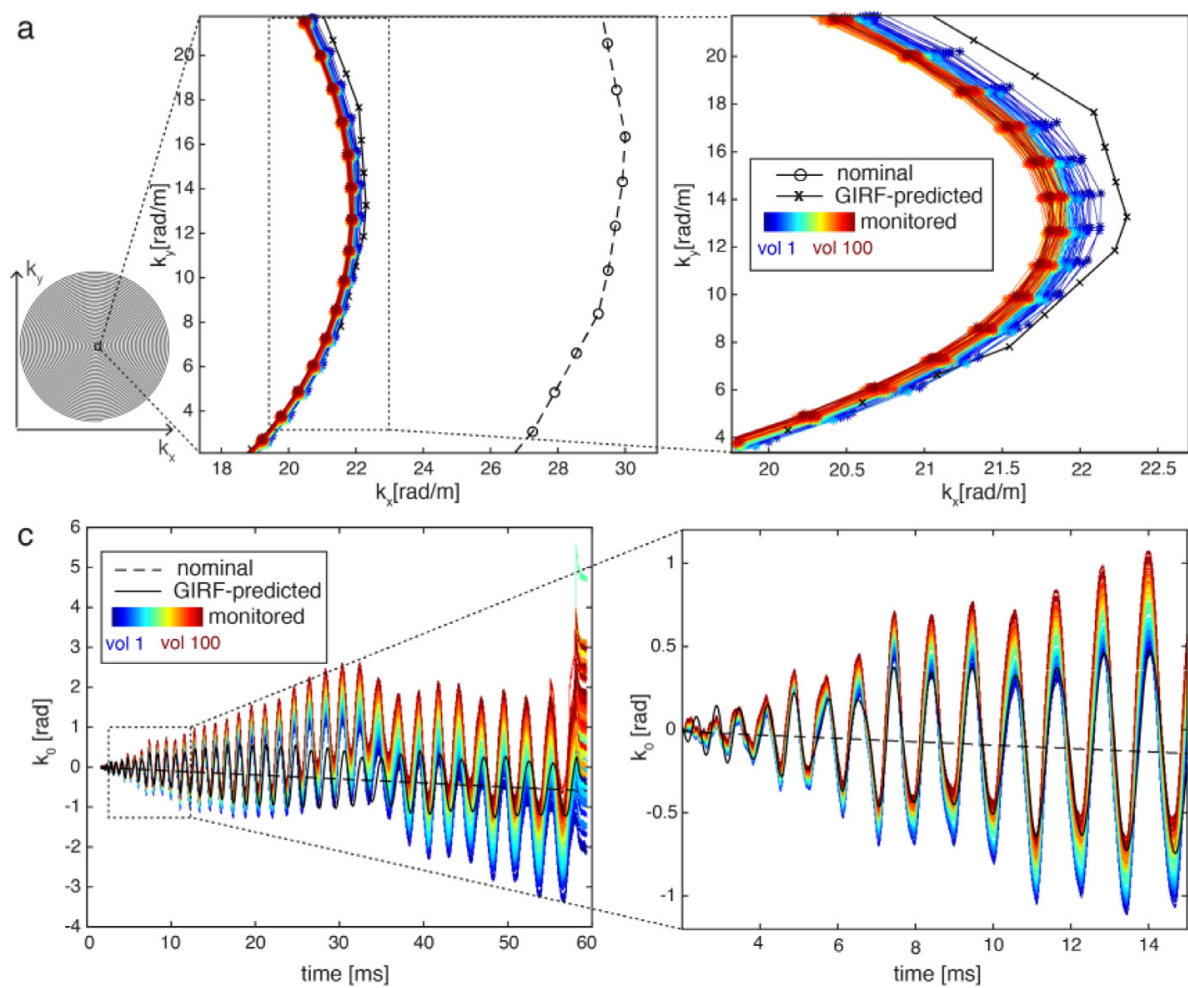

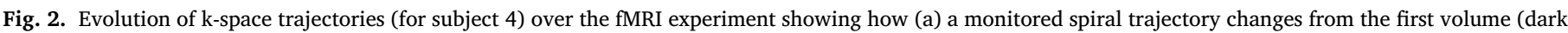

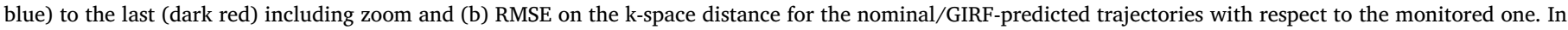
the lower row (c) $k_{0}$ with zoom and (d) RMSE of the nominal/GIRF-predicted $k_{0}$ with respect to the measured one are shown.

The nominal spiral images are heavily corrupted by blurring and geometric distortion (Fig. 3). The GIRF-predicted reconstruction provides much improved image quality. Residual artifacts (mainly subtle blurring and some ringing) can be observed in the difference images to the monitored reconstruction. The global image artifact levels, defined here as the RMSE to the monitored reconstruction averaged over all voxels in a brain mask and all volumes in the fMRI time-series, are reported in Table 1 (image differences reported as percent of the maximum value in the monitored reconstruction). Averaged over all subjects the arti-
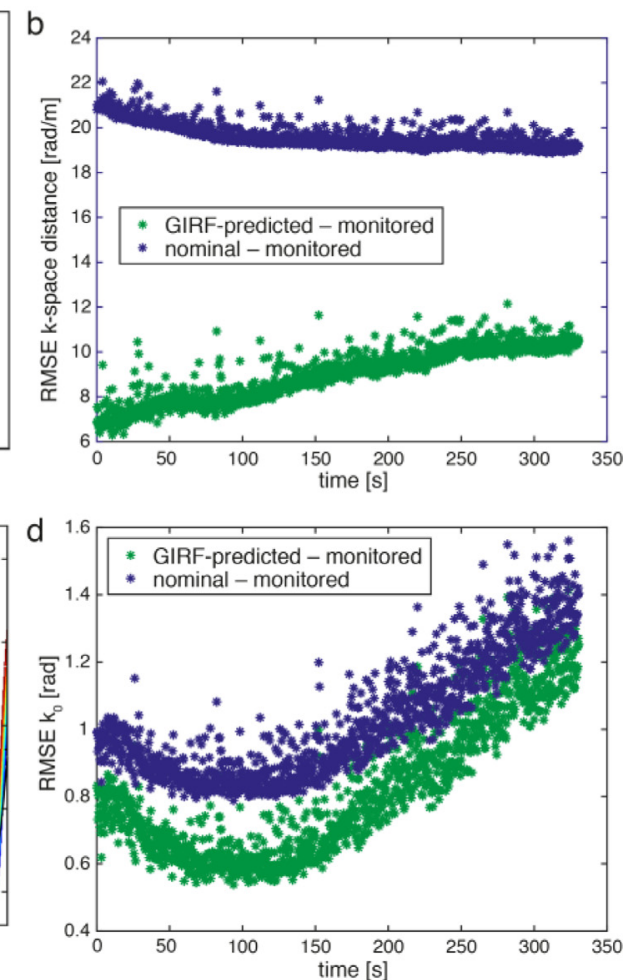


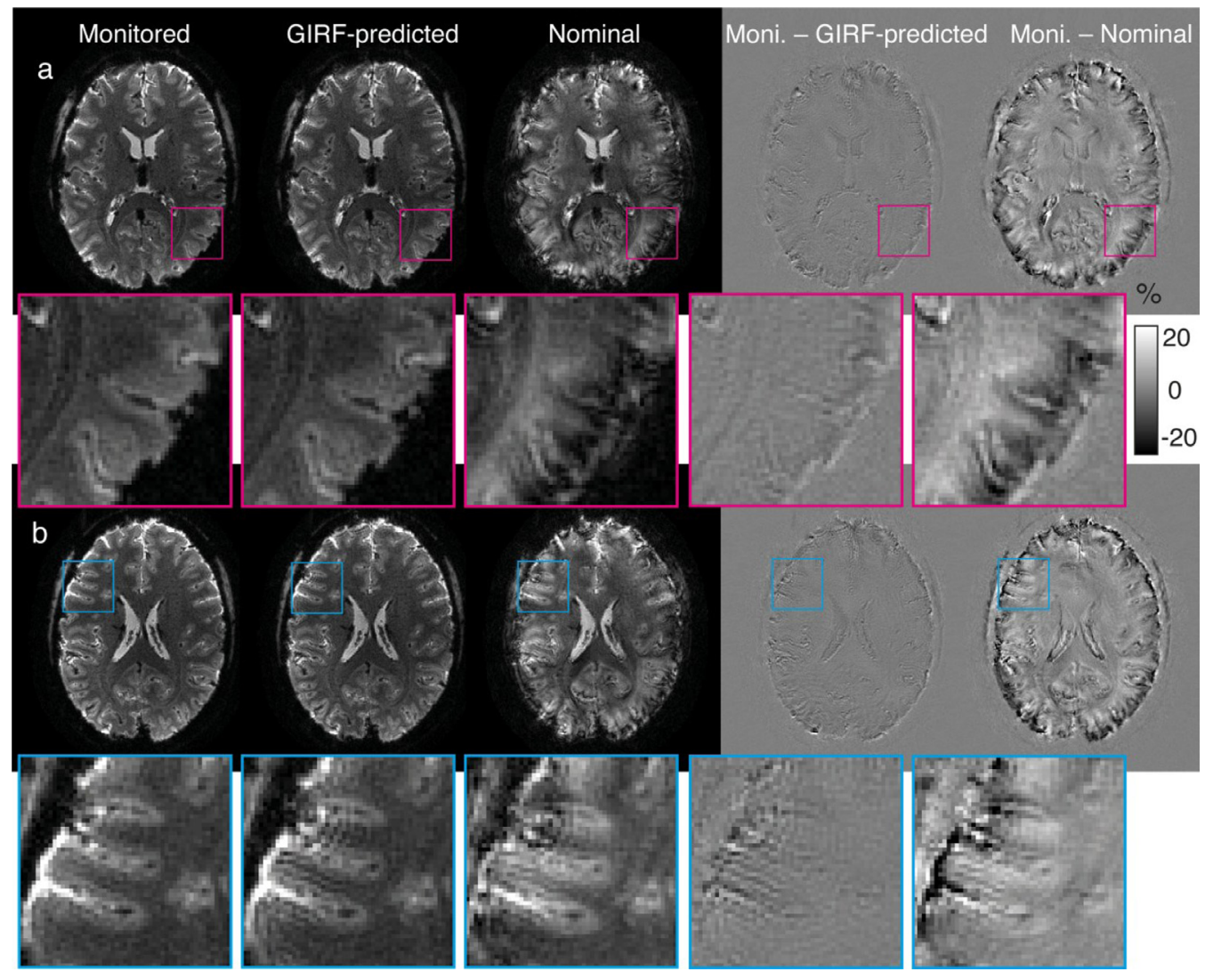

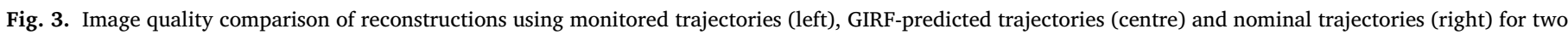

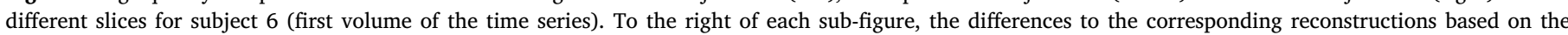

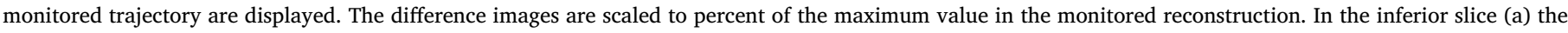

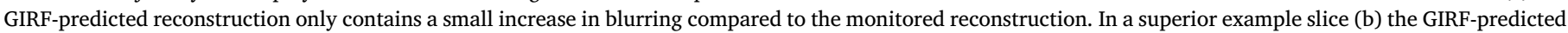
reconstruction additionally exhibits an increase in ringing artifacts.

with an image artifact reduction compared to nominal of $65 \%$ in the first volume and $57 \%$ in the final volume (averaged over all subjects). The change in GIRF-monitored RMSE over the scan aligns well with simultaneously acquired temperature data, which indicates that gradient heating is a driving factor for time-varying gradient system responses not captured by the GIRF.

The average temporal SNR over all subjects (Table 1) is highest for monitored reconstructions, slightly lower for the nominal reconstructions ( $<1 \%$ reduction) and lowest for the reconstructions using GIRFpredicted trajectories ( $\sim 5 \%$ reduction).

Figs. 5 and 6 show fMRI activation maps, in a single subject in various orientations, and in a single slice in all subjects, respectively. For the monitored and GIRF-predicted reconstructions, the spiral fMRI results show good correspondence of the activation with gray matter architecture, while the nominal data contain misplaced activation (apparent for example where activation is crossing white matter boundaries). The activation for the GIRF-predicted and monitored reconstructions largely overlap in all subjects, whereas there are substantial deviations in the nominal activation maps, as demonstrated by activation difference maps (Fig. 6).

The ROC analysis confirms that the GIRF-predicted reconstructions yield nearly as good spatial specificity as the monitored reconstructions, and considerably better than nominal reconstructions (Fig. 7). Compared to the nominal reconstructions, the GIRF reconstructions yield $\sim 122 \%$ increase in specificity as captured by the AUC, averaged over all subjects. The monitored reconstructions in turn provide a smaller additional improvement over the GIRF-reconstructions, with an average increase of the AUC by $\sim 13 \%$. The ROC analysis assumes that all activated voxels within the GM V1-V3 ROI are true positives. Note that this is different to how true positives are defined in the z-statistics map comparison with monitored as the ground truth in Fig. 6.

\section{Discussion}

The main goal of this work was to determine if image reconstruction based on an LTI gradient system model is suitable for use in functional MRI with spiral trajectories. The results presented here first confirmed the conclusion of previous work, showing that reconstructions using the nominal trajectory contain a large amount of artifacts (blurring, ringing and distortion) while monitored and GIRF-predicted trajectories yield high-quality images. Extending from previous work we demonstrated that the image quality of the GIRF-predicted reconstructions remained superior to reconstructions based on nominal trajectories over the course of the time-series. The higher image quality translated into increased spatial specificity in the fMRI analysis, yielding activation patterns that closely followed the gray/white matter architecture in the visual cortex. Nominal reconstructions, on the other hand, yielded misplaced activation that was not localized to the gray matter, due to the blurring and other artifacts present in the images.

We observed that the tSNR was largest for reconstructions using concurrent monitoring. This is expected, as we are reducing the temporal variance in the image time series by correcting for both system-related field variations and physiological field fluctuations (Bollmann et al., 2017; Bright and Murphy, 2017). These are not captured by the GIRF where we use the same trajectory for each volume. We also saw that 

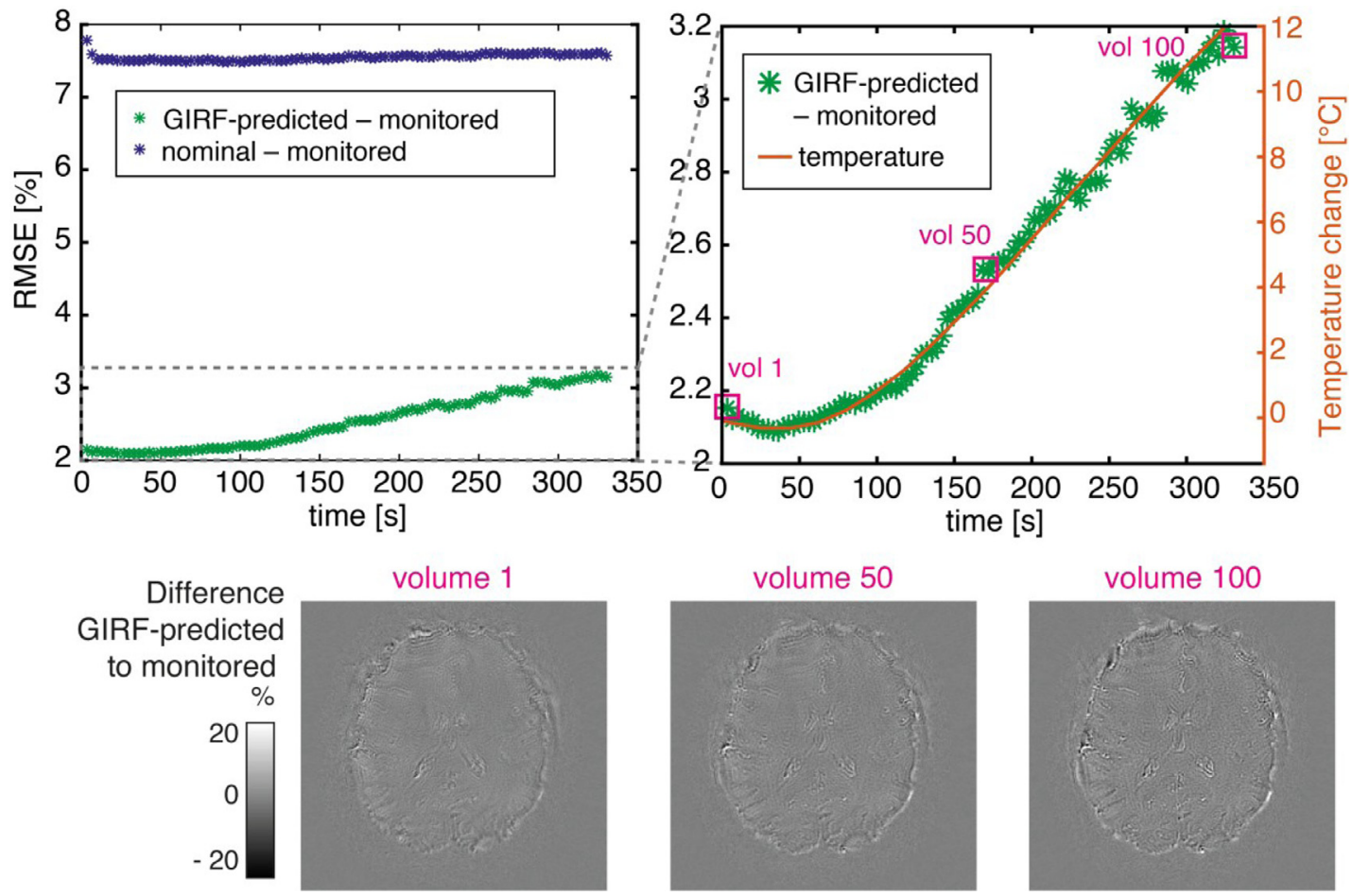

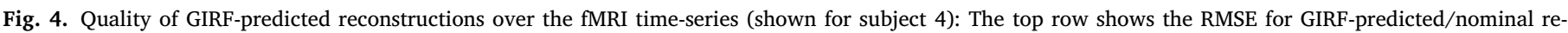

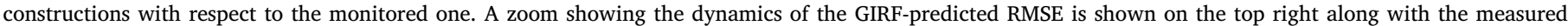

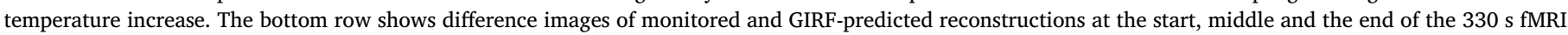
acquisition.

the tSNR is slightly higher for the nominal reconstruction compared to the GIRF reconstruction, which could potentially be explained by the larger amount of blurring present, causing local averaging of signal in the image.

The main source of system-related field variations is heating of the gradient coils and surrounding structures, caused by the long high-duty cycle acquisitions in fMRI. Here, we observed gradient temperature increases of up to $15^{\circ} \mathrm{C}$ over a single $\sim 5$ min fMRI run. Such a substantial temperature increase alters electrical and mechanical material properties and thus changes the behavior of the gradient chain. This is a deviation from the LTI assumption underlying the GIRF approach, which could explain the observed increase in artifacts in the GIRF-predicted reconstructions over the course of the fMRI acquisition. A visual comparison between gradient coil temperature and image RMSE in one subject indicated a close relationship between the two. The GIRF measurement is relatively low duty cycle and was performed starting from a cold state of the system. Therefore, towards the end of an fMRI time series the gradients are in a different thermal state to the one they were characterized in. Overall the impact of this on the fMRI results was small. The spatial specificity of the GIRF-predicted fMRI results was very close to the monitored ones, while it was substantially reduced for the nominal reconstruction. In future work, the GIRF model may be further improved by incorporating temperature-dependent GIRFs (Dietrich et al., 2016b; Nussbaum et al., 2018; Stich et al., 2019). The hardware temperature can easily be assessed via the scanner's temperature monitoring system or using separate temperature sensors and this information can then be used to select the optimal GIRF for each measurement. Alternatively the GIRF approach could be combined with additional navigators that, for example, track global field changes per slice or volume. This would allow adjusting the slope of $\mathrm{k}_{0}$, which we had observed to vary substantially over the course of the fMRI time series (Fig. 2).
There is a complex relationship between the trajectory error and the resulting image quality, which is influenced by many factors not captured by the RMSE. For example, a slow drift and a high-frequency oscillatory component of equally large RMSE will cause very different image artifacts, and the impact will furthermore depend on the underlying trajectory type (e.g. spiral vs. EPI). Results of this and previous work (Vannesjo et al., 2016) suggest that for spiral imaging trajectory deviations near the k-space center are more impactful on the overall image quality than deviations towards the edge of k-space. We also know from previous work that while an oscillatory $\mathrm{k}_{0}$ component has a substantial influence on EPI image quality (Ma et al., 2020; Vannesjo et al., 2016), spiral imaging is more robust to $\mathrm{k}_{0}$ errors, especially when the linear component due to thermal drift is removed (Vannesjo et al., 2016).

The nominal trajectory used in this work included a global delay correction based on minimizing the RMSE to the monitored trajectory. The delay correction of the nominal trajectory did not improve the image quality much, as compared to no delay correction (data not shown). This stands in contrast to EPI where calibrating a delay between odd and even lines typically allows substantial artifact reduction. The delay of the gradient chain is dependent on the frequency of the input waveform, and is therefore not a single parameter valid for all gradient waveforms (Vannesjo et al., 2013). The spiral readout gradients sweep a large range of temporal frequencies, whereas the EPI has a dominant peak at the switching frequency. Presumably for this reason, a single delay correction works well in EPI, whereas for spiral imaging it is important to know the full response over a large range of frequencies.

$\mathrm{B}_{0}$ related artifacts, such as dropout and blurring, scale with field strength, therefore it is especially important to include $\mathrm{B}_{0}$ correction for fMRI at 7T. We observed that the artifacts were worst in the nominal reconstructions near air-tissue interfaces, where $\mathrm{B}_{0}$ inhomogeneity 


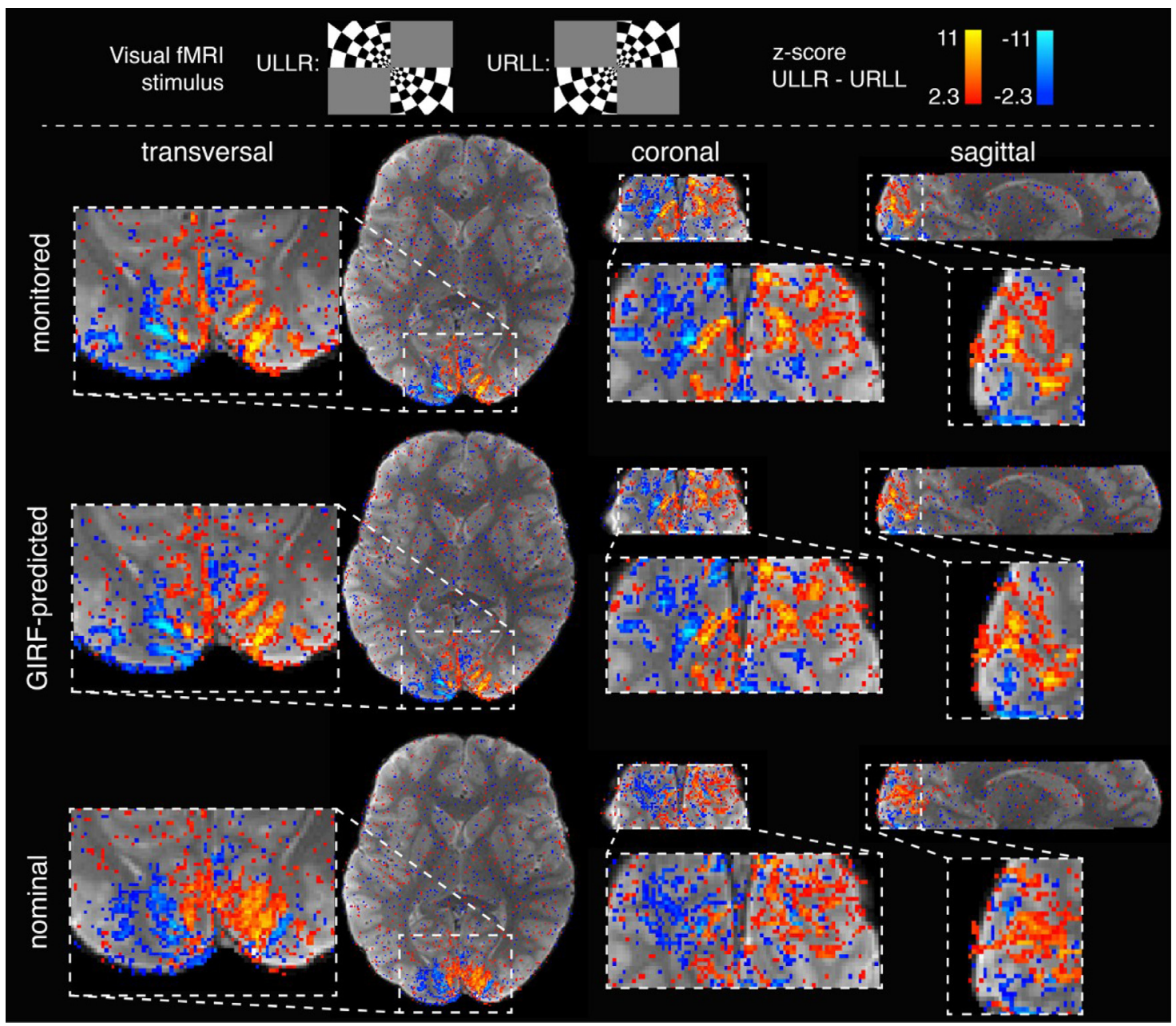

Fig. 5. Evaluation of the fMRI experiment designed to stimulate the quarter-fields of the visual cortex. Z-statistic maps (contrasting ULLR versus URLL) overlaid on the structural image (shown for subject 4). The activation for the monitored and GIRF-predicted reconstructions match the grey matter architecture well, as seen for example along the calcarine sulcus (sagittal view), while the nominal reconstruction results in misplaced activation.

is large, despite the fact that static $\mathrm{B}_{0}$ was accounted for in all reconstructions. This is because $\mathrm{B}_{0}$ correction relies on geometric congruency between the field map and the encoded image (Spirig et al., 2017). Accurate knowledge of the encoding fields therefore becomes even more important at ultra-high field.

We evaluated the spatial specificity of the GIRF-predicted reconstruction, to test suitability of the approach for high-resolution fMRI, as for example required to detect activation on the level of cortical laminae (Huber et al., 2017; Kok et al., 2016) and columns (Cheng et al., 2001; Yacoub et al., 2008). The nominal in-plane resolution of the acquisition was $0.8 \mathrm{~mm}$, but $\mathrm{T}_{2}{ }^{*}$ decay over the readout reduces the effective spatial resolution due to the resulting attenuation of k-space. For our acquisition we therefore expect an effective resolution in the range of 0.94-0.98 mm, based on PSF analysis (Engel et al, 2018). This effect is practically identical for all three reconstructions. In this work we were predominantly interested in the relative spatial specificity of the fMRI activation between the different reconstructions. We assessed this using a ROC-style analysis, which allows quantifying specificity without choosing one reconstruction as a ground truth and is independent of a specific significance threshold. The challenge with this method is that it requires accurate true/false positive masks, which relies on accurate segmentation and registration of an anatomical atlas to the subjects' functional data. To obtain trust-worthy masks, we visually inspected the registration and segmentation in each subject, and chose a conservative threshold for the automatic gray/white matter segmentation. Voxels straddling the border between gray and white matter were therefore not included in either of the masks.

The GIRF-predicted trajectories consistently provided good results for all subjects acquired. There were some inter-subject differences in how closely the GIRF-predicted reconstructions matched the monitored ones (see Figs. 6 and 7). The fMRI scans used in this study were acquired at different time points within the scanning session (sometimes it was the first longer scan of the session whereas other times a few other fMRI runs had been performed immediately beforehand). This could potentially explain some of the differences in performance of the GIRF-prediction between subjects.

The reconstructions using measured trajectories provided the best results, both in terms of image quality and fMRI activation patterns. Concurrent monitoring allows capturing dynamic field effects that violate the LTI assumption, including non-linear and time-dependent responses of the gradient system, as well as non-reproducible effects (e.g. caused by the subject). However, concurrent monitoring is technically challenging and can be difficult to incorporate into routine fMRI scanning. The approach relies on an external hardware setup, optimized for the specific purpose of monitoring long readouts at high resolution. This requires field probes with suitable specifications (e.g. size and doping 


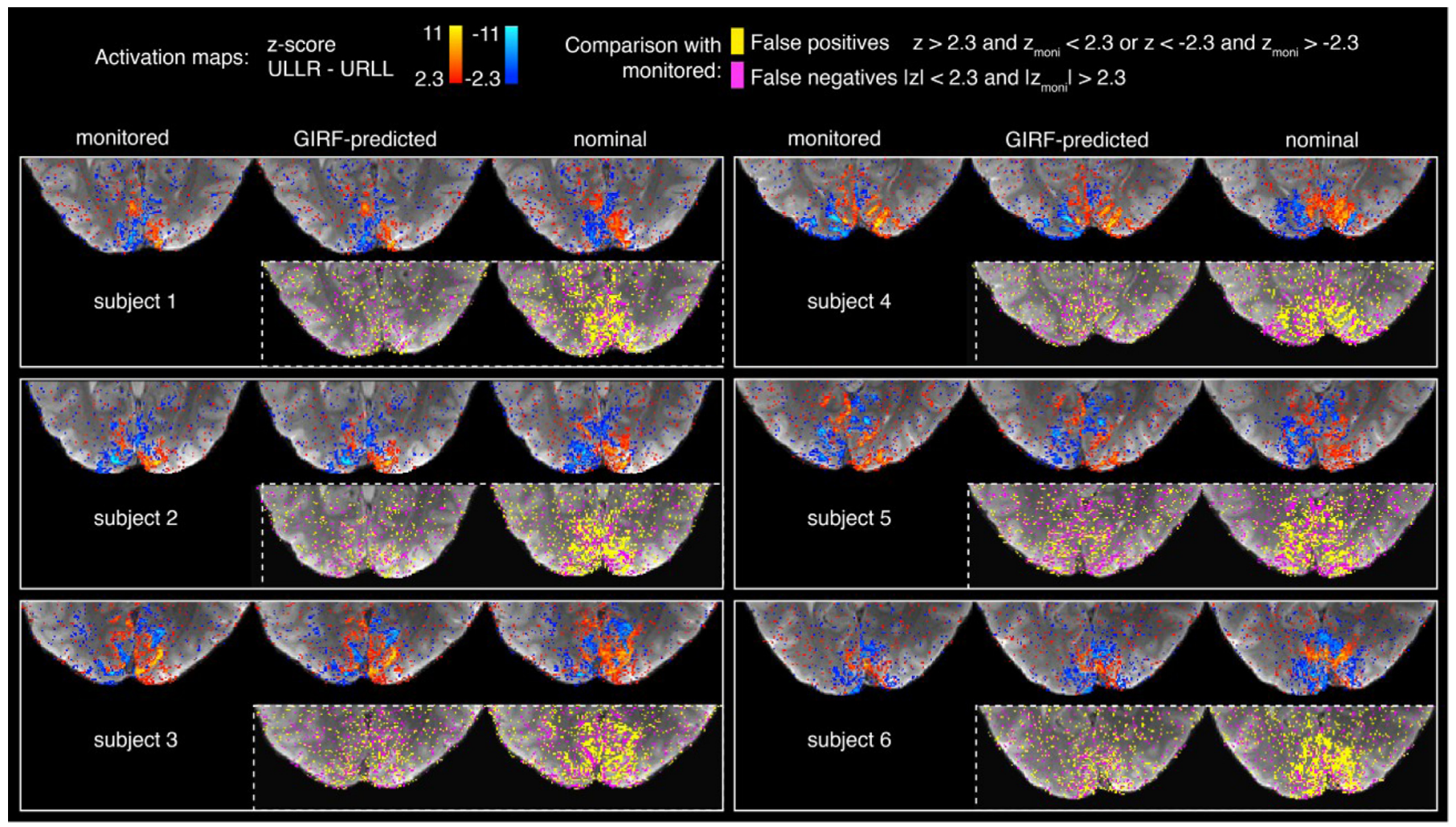

Fig. 6. Activation maps (transversal section of visual cortex) for six subjects. Maps of false positives (yellow) and false negatives (pink) with respect to the monitored reconstruction are displayed below each GIRF-predicted and nominal image.

of the probe) to avoid probe signal de-phasing during the measurement. GIRF-prediction provides an alternative when concurrent monitoring is not feasible, for example if the optimal field monitoring setup is not available or if de-phasing still occurs (e.g., due to a poor shim). Moreover, concurrent monitoring could be combined with the GIRF model, where parts of the readout is measured and the rest is filled in by GIRF prediction (Wilm et al., 2019).

As an alternative to field measurements data-driven methods can be used to compensate for trajectory imperfections without separate trajectory calibration data. This can be achieved by exploiting data redundancy resulting from oversampling the center of k-space (present in many non-Cartesian trajectories) and/or the data redundancy provided my multi-channel acquisitions (Deshmane et al., 2016; Ianni and Grissom, 2016; Jiang et al., 2018; Mani et al., 2017; Wech et al., 2015). The advantage of these methods is that they mostly do not need additional hardware or calibration data, however solving for the image and the trajectory at the same time makes the reconstruction problem more complex and not always well-conditioned.

GIRF characterization is a one-time calibration step (previous work has shown the GIRF to be stable over at least 3 years (Vannesjo et al., 2016)) and can be performed without any specialized equipment. In this study the GIRF was determined using a dynamic field camera, which allows very accurate characterization of the encoding fields with high frequency resolution, including spatial cross-terms and higher-order terms. However the GIRF can also be measured using a phantom-based approach (Addy et al., 2012; Duyn et al., 1998; Rahmer et al., 2019), at the cost of some loss in the frequency resolution of the GIRF (Graedel et al., 2017a).

We used a spiral-out fMRI protocol for this study, but the GIRFbased trajectory prediction is a generic method that can be used for any trajectory. For example it could be employed for hybrid spiralin/out methods (Glover and Law, 2001), which can provide high BOLD sensitivity as well as improved signal dropout artifacts. Beyond spirals the GIRF could enable other non-Cartesian fMRI techniques, which require accurate gradient correction, such as radial (Lee et al., 2010), PROPELLER (Krämer et al., 2012) and TURBINE fMRI (Graedel et al., 2017b). Furthermore, the approach presented here may also be useful to correct EPI trajectories, as the GIRF prediction captures effects that the commonly used odd-even lines EPI Nyquist ghost correction schemes (Schmitt et al., 1998) do not address (Vannesjo et al., 2016).

\section{Conclusion}

GIRF-predicted trajectories have the potential to enable high-quality spiral fMRI in situations where concurrent monitoring is not available. The presented approach requires only a one-time calibration per system, thus the fMRI acquisition is not prolonged or complicated by the acquisition of additional data for correction purposes.

\section{Declaration of Competing Interest}

Bertram J. Wilm is also employed at Skope Magnetic Resonance Technologies Inc. Klaas Pruessmann holds a research agreement with and receives research support from Philips. He is a co-founder and shareholder of Gyrotools Ltd.

\section{Credit authorship contribution statement}

Nadine N. Graedel: Conceptualization, Methodology, Software, Formal analysis, Visualization, Writing - original draft. Lars Kasper: Conceptualization, Methodology, Software, Formal analysis, Investigation, Data curation, Writing - review \& editing. Maria Engel: Methodology, Software, Investigation, Writing - review \& editing. Jennifer Nussbaum: Methodology, Software, Investigation, Writing - review \& editing. Bertram J. Wilm: Methodology, Software, Writing - review \& editing. Klaas P. Pruessmann: Conceptualization, Methodology, Resources, Writing - review \& editing, Funding acquisition. S. Johanna 

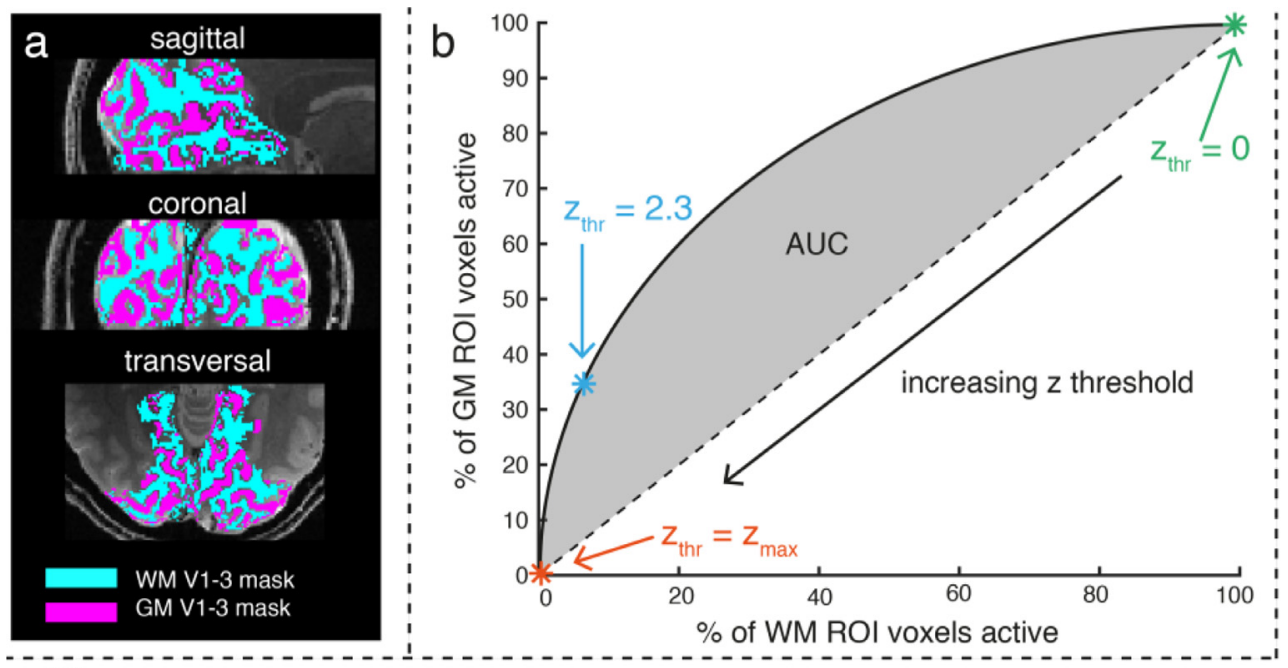

C ROC curves for all six subjects

- monitored

- GIRF-predicted

- nominal

- $z_{\text {thr }}=2.3$ monitored

* $z_{\text {thr }}=2.3$ GIRF-predicted

$+z_{\text {thr }}=2.3$ nominal

- - - line of no-discrimination
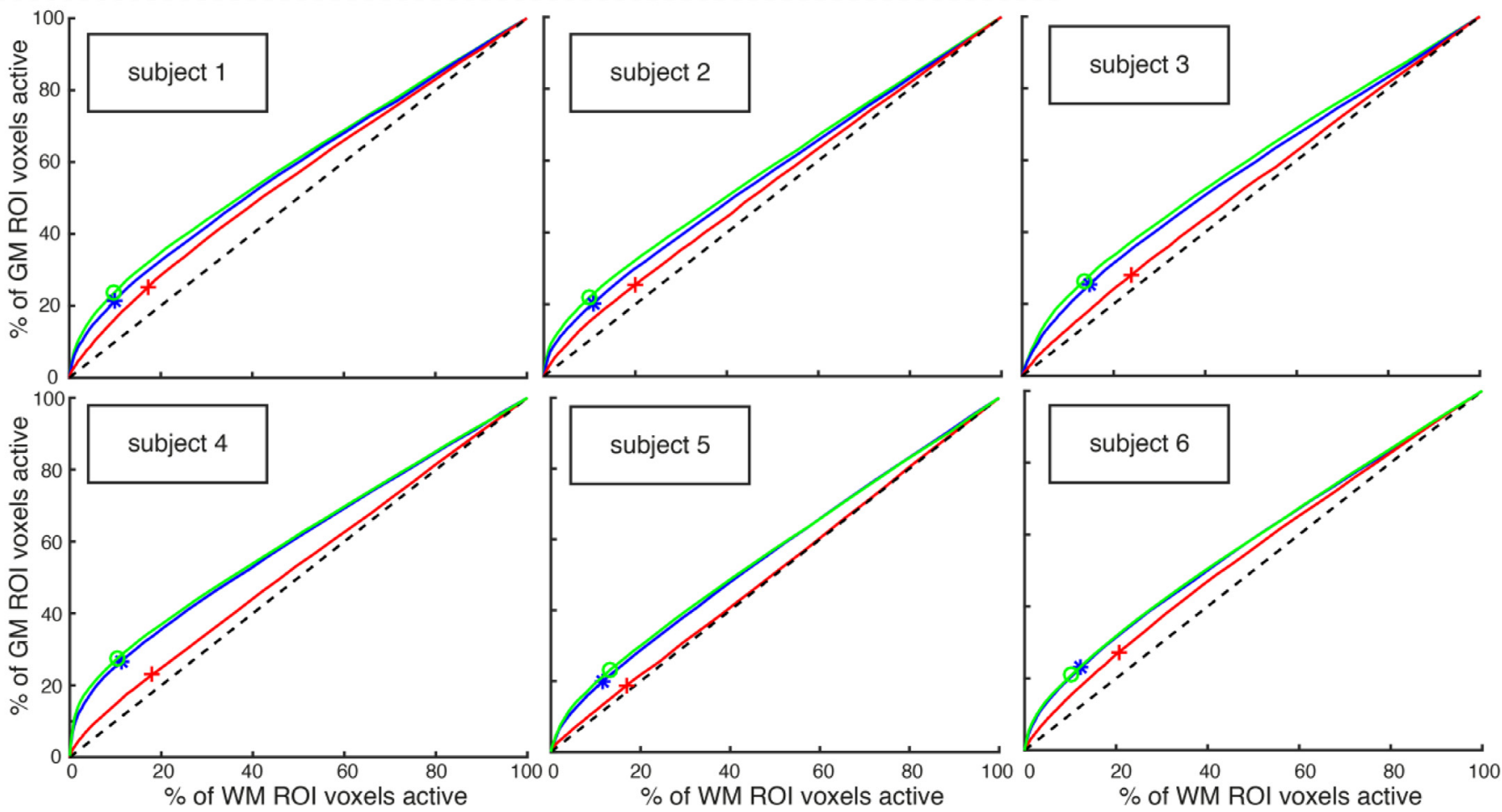

Fig. 7. (a) Grey and white matter masks of V1-V3 used for the analysis. (b) Schematic explaining receiver operating characteristic (ROC) curve analysis used to assess the spatial specificity of the different reconstructions (without having to choose a specific one as ground truth). The dashed line is the line of no discrimination, indicating equal amounts of true and false positives. The area under the curves (AUC) values were used as a summary metric and are reported in Table 1. (c) ROC curves for all six subjects, indicating that the spatial specificity of the activation is highest for the monitored reconstructions. The GIRF-predicted reconstructions result in only slightly reduced specificity whereas the nominal curve lies substantially closer to the line of no discrimination.

Vannesjo: Conceptualization, Methodology, Software, Resources, Writing - review \& editing, Project administration, Supervision, Funding acquisition.

\section{Acknowledgments}

We would like to thank the Oxford-Brain@McGill-ZNZ Partnership in the Neurosciences for funding this project (OMZPN/2015/1/3). This work was also supported by the Wellcome Trust (Senior Research Fellowship 202788/Z/16/Z), the European Union's Horizon 2020 research and innovation programme under the Marie Sklodowska-Curie grant agreement No 659263 (J.V.) and the NCCR "Neural Plasticity and Repair" (L.K.). The Wellcome Centre for Integrative Neuroimaging is sup- ported by core funding from the Wellcome Trust (203139/Z/16/Z). Technical support from Philips Healthcare is gratefully acknowledged.

\section{Code and data availability}

The data from subject 6 will be made available on ETH Research Collection upon publication. This will include the raw coil data in ISMRMRD format including the field monitoring data for the entire fMRI run the data collection doi:10.3929/ethz-b-000487412 associated with Kasper et al. (2019). In a second data collection doi:10.3929/ethzb-000510127 specific to this publication we include ISMRMD format data of volumes 1, 50 and 100 with monitored, GIRF-predicted and nominal trajectories. Additionally, we include the reconstructed image time series using the nominal, GIRF-predicted and monitored k- 
space trajectories for subject 6 . The raw data sets from the other subjects cannot be made publicly available, as we did not obtain explicit subject consent to share data for these subjects. However, we provide the mean spiral fMRI images for all three reconstructions with the corresponding activation maps for all subjects on NeuroVault (https://identifiers.org/neurovault.collection:6526).

The image reconstruction in this work was performed using custom Matlab implementation of CG-SENSE (Pruessmann et al., 2001) algorithm. A demo version of the reconstruction pipeline is publicly available on GitHub (https://github.com/mrtm-zurich/ rrsg-arbitrary-sense), however without the multi-frequency interpolation used for the $\mathrm{B}_{0}$ correction in this work.

Scripts (bash and Matlab) for the post-processing and analysis pipeline will be available on https://github.com/MRI-gradient/ paper-GIRF-spiral-fMRI. Matlab code for GIRF calculation and trajectory prediction using the GIRF can also be found in the same repository.

\section{Supplementary materials}

Supplementary material associated with this article can be found, in the online version, at doi:10.1016/j.neuroimage.2021.118674.

\section{References}

Addy, N.O., Wu, H.H., Nishimura, D.G., 2012. Simple method for MR gradient system characterization and k-space trajectory estimation. Magn. Reson. Med. 68, 120-129.

Ahn, C.B., Kim, J.H., Cho, Z.H., 1986. High-Speed Spiral-Scan Echo Planar NMR Imaging-I. IEEE Trans. Med. Imaging. doi:10.1109/TMI.1986.4307732.

Andersson, J.L.R., Skare, S., Ashburner, J., 2003. How to correct susceptibility distortions in spin-echo echo-planar images: application to diffusion tensor imaging. Neuroimage 20, $870-888$.

Barmet, C., De Zanche, N., Pruessmann, K.P., 2008. Spatiotemporal magnetic field monitoring for MR. Magn. Reson. Med. 60, 187-197.

Bhavsar, P.S., Zwart, N.R., Pipe, J.G., 2014. Fast, variable system delay correction for spiral MRI. Magn. Reson. Med. doi:10.1002/mrm.24730.

Bollmann, S., Kasper, L., Vannesjo, S.J., Diaconescu, A.O., Dietrich, B.E., Gross, S., Stephan, K.E., Pruessmann, K.P., 2017. Analysis and correction of field fluctuations in fMRI data using field monitoring. Neuroimage doi:10.1016/j.neuroimage.2017.01.014.

Börnert, P., Schomberg, H., Aldefeld, B., Groen, J., 1999. Improvements in spiral MR imaging. Magn. Reson. Mater. Phys. Biol. Med. 9, 29-41.

Bright, M.G., Murphy, K., 2017. Cleaning up the fMRI time series: mitigating noise with advanced acquisition and correction strategies. Neuroimage doi:10.1016/j.neuroimage.2017.03.056

Campbell-Washburn, A.E., Xue, H., Lederman, R.J., Faranesh, A.Z., Hansen, M.S., 2016. Real-time distortion correction of spiral and echo planar images using the gradient system impulse response function. Magn. Reson. Med. doi:10.1002/mrm.25788.

Cheng, K., Waggoner, R.A., Tanaka, K., 2001. Human ocular dominance columns as revealed by high-field functional magnetic resonance imaging. Neuron doi:10.1016/S0896-6273(01)00477-9.

De Zanche, N., Barmet, C., Nordmeyer-Massner, J.A., Pruessmann, K.P., 2008. NMR probes for measuring magnetic fields and field dynamics in MR systems. Magn. Reson. Med. 60, 176-186.

Deshmane, A., Blaimer, M., Breuer, F., Jakob, P., Duerk, J., Seiberlich, N., Griswold, M., 2016. Self-calibrated trajectory estimation and signal correction method for robust radial imaging using GRAPPA operator gridding. Magn. Reson. Med. 75, 883-896.

Dietrich, B.E., Brunner, D.O., Wilm, B.J., Barmet, C., Gross, S., Kasper, L., Haeberlin, M., Schmid, T., Vannesjo, S.J., Pruessmann, K.P., 2016a. A field camera for MR sequence monitoring and system analysis. Magn. Reson. Med. 75, 1831-1840.

Dietrich, B.E., Reber, J., Brunner, D.O., Wilm, B.J., Pruessmann, K.P., 2016b. Analysis and prediction of gradient response functions under thermal load. In: Proceedings of the International Society for Magnetic Resonance in Medicine, Singapore, p. 3551.

Duyn, J.H., Yang, Y., Frank, J.A., van der Veen, J.W., 1998. Simple correction method for k-space trajectory deviations in MRI. J. Magn. Reson. 132, 150-153.

Engel, M., Kasper, L., Barmet, C., Schmid, T., Vionnet, L., Wilm, B., Pruessmann, K.P., 2018. Single-shot spiral imaging at 7 T. Magn. Reson. Med. 80, 1836-1846.

Glover, G.H., 2012. Spiral imaging in fMRI. Neuroimage 62, 706-712.

Glover, G.H., Law, C.S., 2001. Spiral-in/out BOLD fMRI for increased SNR and reduced susceptibility artifacts $46,515-522$.

Glover, G.H., Lee, A.T., 1995. Motion artifacts in fMRI: comparison of 2DFT with PR and spiral scan methods. Magn. Reson. Med. 33, 624-635.

Graedel, N.N., Hurley, S.A., Clare, S., Miller, K.L., Pruessmann, K.P., Vannesjo, S.J., 2017a. Comparison of gradient impulse response functions measured with a dynamic field camera and a phantom-based technique. In: Proceedings of the ESMRMB, p. 378.

Graedel, N.N., McNab, J.A., Chiew, M., Miller, K.L., 2017b. Motion correction for functional MRI with three-dimensional hybrid radial-Cartesian EPI. Magn. Reson. Med. 78, 527-540. doi:10.1002/mrm.26390.

Huber, L., Handwerker, D.A., Jangraw, D.C., Chen, G., Hall, A., Stüber, C., GonzalezCastillo, J., Ivanov, D., Marrett, S., Guidi, M., Goense, J., Poser, B.A., Ban- dettini, P.A., 2017. High-resolution CBV-fMRI allows mapping of laminar activity and connectivity of cortical input and output in human M1. Neuron doi:10.1016/j.neuron.2017.11.005.

Ianni, J.D., Grissom, W.A., 2016. Trajectory auto-corrected image reconstruction. Magn. Reson. Med. doi:10.1002/mrm.25916.

Jenkinson, M., Bannister, P., Brady, M., Smith, S., 2002. Improved optimization for the robust and accurate linear registration and motion correction of brain images. Neuroimage $17,825-841$.

Jenkinson, M., Beckmann, C.F., Behrens, T.E.J., Woolrich, M.W., Smith, S.M., 2012. FSL. Neuroimage 62, 782-790.

Jiang, W., Larson, P.E.Z., Lustig, M., 2018. Simultaneous auto-calibration and gradient delays estimation (SAGE) in non-Cartesian parallel MRI using low-rank constraints. Magn. Reson. Med. doi:10.1002/mrm.27168.

Kaldoudi, E., Williams, S.C.R., Barker, G.J., Tofts, P.S., 1993. A chemical shift selective inversion recovery sequence for fat-suppressed MRI: theory and experimental validation. Magn. Reson. Imaging. doi:10.1016/0730-725X(93)90067-N.

Kasper, L., Engel, M., Barmet, C., Häberlin, M., Wilm, B.J., Dietrich, B.E., Schmid, T., Gross, S., Brunner, D.O., Stephan, K.E., Pruessmann, K.P., 2018. Rapid anatomical brain imaging using spiral acquisition and an expanded signal model. Neuroimage 168, 88-100.

Kasper, L., Engel, M., Heinzle, J., Mueller-Schrader, M., Jonas Reber, T.S., Barmet, C., Wilm, B.J., Stephan, K.E., Pruessmann, K.P., 2019. Advances in spiral fMRI: a high-resolution study with single-shot acquisition. NeuroImage doi:10.1016/j.neuroimage.2021.118738.

Kasper, L., Häberlin, M., Dietrich, B.E., Gross, S., Barmet, C., Wilm, B.J., Vannesjo, S.J., Brunner, D.O., Ruff, C.C., Stephan, K.E., Pruessmann, K.P., 2014. Matched-filter acquisition for BOLD fMRI. Neuroimage 100, 145-160.

Kok, P., Bains, L.J., Van Mourik, T., Norris, D.G., De Lange, F.P., 2016. Selective activation of the deep layers of the human primary visual cortex by top-down feedback. Curr. Biol. doi:10.1016/j.cub.2015.12.038.

Krämer, M., Jochimsen, T.H., Reichenbach, J.R., 2012. Functional magnetic resonance imaging using PROPELLER-EPI. Magn. Reson. Med. 68, 140-151.

Lee, G.R., Griswold, M.A., Tkach, J.A., 2010. Rapid 3D radial multi-echo functional magnetic resonance imaging. Neuroimage 52, 1428-1443.

Lustig, M., Kim, S.-J., Pauly, J.M., 2008. A fast method for designing time-optimal gradient waveforms for arbitrary k-space trajectories. IEEE Trans. Med. Imaging 27, 866-873.

Ma, R., Akçakaya, M., Moeller, S., Auerbach, E., Uğurbil, K., Van de Moortele, P.F., 2020. A field-monitoring-based approach for correcting eddy-current-induced artifacts of up to the 2nd spatial order in human-connectome-project-style multiband diffusion MRI experiment at 7T: A pilot study. Neuroimage doi:10.1016/j.neuroimage.2020.116861.

Man, L.C., Pauly, J.M., Macovski, A., 1997. Multifrequency interpolation for fast offresonance correction. Magn. Reson. Med. doi:10.1002/mrm.1910370523.

Mani, M., Jacob, M., Kelley, D., Magnotta, V., 2017. Multi-shot sensitivity-encoded diffusion data recovery using structured low-rank matrix completion (MUSSELS). Magn. Reson. Med. doi:10.1002/mrm.26382.

Noll, D.C., Cohen, J.D., Meyer, C.H., Schneider, W., 1995. Spiral K-space MR imaging of cortical activation. J. Magn. Reson. Imaging 5, 49-56.

Nussbaum, J., Wilm, B.J., Dietrich, B.E., Pruessmann, K.P., 2018. IMPROVED THERMAL MODELLING AND PREDICTION OF GRADIENT RESPONSE USING SENSOR PLACEMENT GUIDED BY INFRARED PHOTOGRAPHY. In: Proceedings of the International Society for Magnetic Resonance in Medicine, Paris, p. 4210.

Pruessmann, K.P., Weiger, M., Börnert, P., Boesiger, P., 2001. Advances in sensitivity encoding with arbitrary k-space trajectories. Magn. Reson. Med. 46, 638-651.

Rahmer, J., Mazurkewitz, P., Börnert, P., Nielsen, T., 2019. Rapid acquisition of the 3D MRI gradient impulse response function using a simple phantom measurement. Magn. Reson. Med. doi:10.1002/mrm.27902.

Robison, R.K., Devaraj, A., Pipe, J.G., 2010. Fast, simple gradient delay estimation for spiral MRI. Magn. Reson. Med. 63, 1683-1690.

Schleicher, A., Palomero-Gallagher, N., Morosan, P., Eickhoff, S.B., Kowalski, T., de Vos, K., Amunts, K., Zilles, K., 2005. Quantitative architectural analysis: a new approach to cortical mapping. Anat. Embryol. 210, 373-386 (Berl).

Schmitt, F., Stehling, M.K., Turner, R., 1998. Echo-PlanAR IMAGING, THEORY, TechNIQUE AND APPLICATION. Springer.

Smith, S.M., Jenkinson, M., Woolrich, M.W., Beckmann, C.F., Behrens, T.E.J., Johansen-Berg, H., Bannister, P.R., De Luca, M., Drobnjak, I., Flitney, D.E., Niazy, R.K., Saunders, J., Vickers, J., Zhang, Y., De Stefano, N., Brady, J.M., Matthews, P.M., 2004. Advances in functional and structural MR image analysis and implementation as FSL. Neuroimage 23 (Suppl 1), S208-S219.

Spirig, Y., Graedel, N.N., Kasper, L., Miller, K.L., Frost, R., Clare, S., Pruessmann, K.P., Vannesjo, S.J., 2017. Interaction between trajectory deviations and B0 field inhomogeneity in readout-segmented EPI and spiral imaging. In: Proceedings of the International Society for Magnetic Resonance in Medicine, p. 3917.

Stich, M., Pfaff, C., Wech, T., Slawig, A., Ruyters, G., Dewdney, A., Ringler, R., Köstler, H., 2019. Temperature-dependent gradient system response. Magn. Reson. Med. 44, 532

Sutton, B.P., Noll, D.C., Fessler, J.A., 2003. Fast, Iterative Image Reconstruction for MRI in the Presence of Field Inhomogeneities. IEEE Trans. Med. Imaging 22, 178-188.

Vannesjo, S.J., Graedel, N.N., Kasper, L., Gross, S., Busch, J., Haeberlin, M., Barmet, C., Pruessmann, K.P., 2016. Image reconstruction using a gradient impulse response model for trajectory prediction. Magn. Reson. Med. doi:10.1002/mrm.25841.

Vannesjo, S.J., Haeberlin, M., Kasper, L., Pavan, M., Wilm, B.J., Barmet, C., Pruessmann, K.P., 2013. Gradient system characterization by impulse response measurements with a dynamic field camera. Magn. Reson. Med. 69, 583-593.

Warnking, J., Dojat, M., Guérin-Dugué, A., Delon-Martin, C., Olympieff, S., Richard, N., Chéhikian, A., Segebarth, C., 2002. fMRI retinotopic mapping-step by step. Neuroimage $17,1665-1683$. 
Wech, T., Tran-Gia, J., Bley, T.A., Köstler, H., 2015. Using self-consistency for an iterative trajectory adjustment (SCITA). Magn. Reson. Med. doi:10.1002/mrm.25244.

Wilm, B.J., Barmet, C. Gross, S., Kasper, L., Vannesjo, S.J., Haeberlin, M., Dietrich, B.E., Brunner, D.O., Schmid, T., Pruessmann, K.P., 2016. Single-shot spiral imaging enabled by an expanded encoding model: Demonstration in diffusion MRI. Magn. Reson. Med. 77, 83-91.

Wilm, B.J., Dietrich, B.E., Reber, J., Vannesjo, S.J., Pruessmann, K.P., 2019. Gradient response harvesting for continuous system characterization during MR sequences. IEEE Trans. Med. Imaging 1.

Woolrich, M.W., Ripley, B.D., Brady, M., Smith, S.M., 2001. Temporal autocorrelation in univariate linear modeling of FMRI data. Neuroimage doi:10.1006/nimg.2001.0931.

Wright, K.L., Hamilton, J.I., Griswold, M.A., Gulani, V., Seiberlich, N., 2014. Non-Cartesian parallel imaging reconstruction. J. Magn. Reson. Imaging 40, 1022-1040.
Yacoub, E., Harel, N., Ugurbil, K., 2008. High-field fMRI unveils orientation columns in humans. Proc. Natl. Acad. Sci. U. S. A. 105, 10607-10612.

Yang, Y., Glover, G.H., van Gelderen, P., Patel, A.C., Mattay, V.S., Frank, J.A., Duyn, J.H., 1998. A comparison of fast MR scan techniques for cerebral activation studies at 1.5 tesla. Magn. Reson. Med. 39, 61-67.

Zhang, Y., 0001, M.B., Smith, S.M., 2001. Segmentation of brain mr images through a hidden markov random field model and the expectation maximization algorithm. IEEE Trans. Med. Imaging 20, 45-57.

Zilles, K., Amunts, K., 2010. Centenary of Brodmann's map-conception and fate. Nat. Rev. Neurosci. 11, 139-145. 